\title{
Food neophobia and its relationship with dietary variety and quality in Irish adults: findings from a national cross-sectional study
}

\author{
D. Hazley ${ }^{1}$, S. McCarthy ${ }^{2}$, M. Stack ${ }^{1}$, B.A. McNulty ${ }^{3}$, J. Walton ${ }^{4}$, A. Flynn ${ }^{5}$ and J.M. Kearney ${ }^{1}$ \\ ${ }^{1}$ School of Biological \& Health Sciences, Technological University Dublin, Dublin, Ireland, \\ ${ }^{2}$ Teagasc Food Research Centre, Ashtown, Dublin, Ireland, \\ ${ }^{3}$ School of Agriculture and Food Science, UCD Institute of Food and Health, University College Dublin, \\ Dublin, Ireland, \\ ${ }^{4}$ Department of Biological Science, Munster Technological University, Cork, Ireland and \\ ${ }^{5}$ School of Food and Nutritional Sciences, University College Cork, Cork, Ireland
}

Food neophobia is characterised by a reluctance to eat novel or unfamiliar foods and has been linked to reduced dietary variety and quality, predominantly in children ${ }^{(1)}$. This study aimed to explore the relationship between food neophobia and dietary variety and quality in a nationally representative sample of Irish adults.

This study was conducted using a sub-sample of the National Adults Nutrition Survey collected between $2008-2010(n=1088$, male/female, 539/549). Food and nutrient intakes were assessed using a 4-day semi-weighted food diary. Food neophobia was measured using the Food Neophobia Scale (FNS). Dietary variety was assessed in three ways; Total Dietary Variety (TDV), Food-Group Variety (FGV) and Fruit and Vegetable Variety (FVV). Diet quality was assessed using the Mean Adequacy Ratio (MAR) and Nutrient-Rich Food Index (NRF9.3). Multiple linear regressions were used to assess the linear relationships between a 10-point increase in FNS score $\left(\beta_{10}\right)$ and all dietary measures (dietary variety, diet quality, fruit and vegetable intake, 10 macronutrients and 19 micronutrients), controlling for age, gender, education level, social class, location and BMI. Under-reporters $(n=370)$ were defined as individuals with an energy intake to basal metabolic rate ratio (EI: BMR) of less than $1.1^{(2)}$. All analyses were run with and without under-reporters included. $P<0.001$ was considered statistically significant after adjustment for multiple comparisons using the Bonferroni correction.

The mean FNS (possible range 10-70) for the sample population was 34.35 ( $\mathrm{SD}=13.33$ ). When under-reporters were excluded, FNS was found to be inversely associated with TDV $\left(\beta_{10}=-1.228 ; 95 \% \mathrm{CI}:-1.714 ;-0.742 ; P<0.001\right), \mathrm{FGV}\left(\beta_{10}=-0.359 ; 95 \%\right.$ CI: $-0.503 ;-0.215 ; P<0.001)$ and FVV $\left(\beta_{10}=-0.544 ; 95 \% \mathrm{CI}:-0.767 ;-0.321 ; P<0.001\right)$. In addition, FNS was negatively associated with vitamin $C\left(\beta_{10}=-5.238 ; 95 \%\right.$ CI: $\left.-8.360 ;-2.116 ; P=0.001\right)$, magnesium $\left(\beta_{10}=-8.822 ; 95 \%\right.$ CI: -13.812 ; $-3.832 ; P<0.001)$ and fruit and vegetable intakes $\left(\beta_{10}=-26.334 ; 95 \% \mathrm{CI}:-36.325 ;-16.342 ; P<0.001\right)$ and positively associated with percentage energy from free sugars $\left(\beta_{10}=0.574 ; 95 \% \mathrm{CI}: 0.288 ; 0.859 ; P<0.001\right)$. However, FNS was not significantly associated with any other nutrients, MAR and NRF9.3. Similar relationships were observed when under-reporters were included.

These findings provide further evidence that higher food neophobia is associated with a lower dietary variety, as well as reduced intakes of vitamin C, magnesium and fruit and vegetable intake and an increased intake of free sugars. However, food neophobia was not significantly associated with overall diet quality as has been observed in other studies ${ }^{(3)}$. While these results suggest food neophobia may not be a particularly important risk factor for poor nutrient status, overall adherence to dietary recommendations remains low within the Irish population and food neophobia may further inhibit the adaption of healthy and sustainable diets. Future research should seek to understand the implications of food neophobia on dietary behaviour change.

\section{References}

1. Dovey TM, Staples PA, Gibson EL et al. (2008) Appetite. 50, 181-193.

2. McGowan M, Harrington K, Kiely M et al. (2001) Public Health Nutr. 4, 1043-1050.

3. Rabadán A \& Bernabéu R (2021). Food Qual. Prefer. 93, 104-241. 